
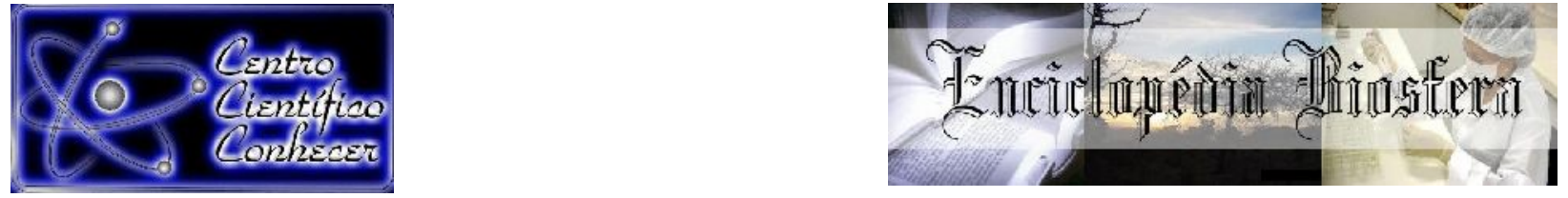

\title{
DISTRIBUIÇÃO DA PRECIPITAÇÃO E AJUSTE DE MODELOS PROBABILÍSTICOS PARA FORMOSO, MG
}

\author{
Rômulo Gustavo de Carvalho Silva ${ }^{1}$ Alexandre Martins Abdão dos Passos ${ }^{2}$ Andreia \\ Marcilane Aker ${ }^{3}$ Victor Almeida Cordeiro ${ }^{4}$ Camila Barbosa Alves ${ }^{5}$.
}

1 Graduando de Engenharia Agronômica, Universidade Federal de São João del-Rei Campus CSL, Sete Lagoas - MG, Brasil.

2 Pesquisador da Embrapa Milho e Sorgo, Sete Lagoas, MG, Brasil. E-mail:

(alexandre.abdao@embrapa.br)

3 Doutoranda em Produção Vegetal (UFVJM), Diamantina, MG, Brasil.

4 Graduando de Engenharia Agronômica, Universidade Federal de São João del-Rei

Campus CSL, Sete Lagoas - MG, Brasil.

5 Graduanda de Engenharia Florestal, Universidade Federal de São João del-Rei

Campus CSL, Sete Lagoas - MG, Brasil.

Recebido em: 04/10/2019 - Aprovado em: 30/11/2019 - Publicado em: 15/12/2019

DOI: 10.18677/EnciBio_2019B23

\begin{abstract}
RESUMO
A precipitação é um fator decisivo no planejamento de ações na agricultura. Conhecer a distribuição de chuvas é ferramenta que auxilia de forma incisiva na tomada de decisões e quando aliado a técnicas conservacionistas de solo e água aumentam a efetividade das atividades principalmente em sistemas produtivos de sequeiro. Com o objetivo de conhecer a distribuição da precipitação e o ajuste de modelos probabilísticos, o presente estudo avaliou historicamente os dados da cidade de Formoso, em Minas Gerais. Os dados de precipitação foram obtidos de uma série histórica de 33 anos, captados de uma estação climática automática. Observou-se que os meses de maior volume de chuva abrange o período de novembro a fevereiro, que compreende um acumulado de $783 \mathrm{~mm}$, volume suficiente para o cultivo de diversas culturas de verão. O período de menor precipitação, entre abril e outubro, apresentou uma média de apenas $32 \mathrm{~mm}$ ao mês. Os melhores ajustes de modelos aos volumes de precipitação foram observados no gama e weibull.
\end{abstract}

PALAVRAS-CHAVE: Modelos; Pluviometria; Precipitação.

\section{DISTRIBUTION OF PRECIPITATION AND MODELS OF PROBABILITY FOR FORMOSO CITY, MG}

\section{ABSTRACT}

Precipitation is a decisive factor in the planning of actions in agriculture, knowing its distribution is a tool that assists incisively in decision-making. Knowing rainfall combined with conservation techniques increases the effectiveness of activities, especially in non-irrigated systems. To know the distribution of precipitation and the adjustment of probabilistic models, the present study evaluated historically 33 years 
of an automatic climatic station data in the city of Formoso, Minas Gerais State. It was observed as rainy months the period from November to February, with a total of $783 \mathrm{~mm}$ in the season. This amount is enough to produce a larger range of crops. The drier period with low rates of precipitation, since April to October, presented an average of $32 \mathrm{~mm}$ per month. We fitted as the best models, the gamma and weibull models to the monthly volumetric data.

KEYWORDS: Models; Rainfall; Precipitation.

\section{INTRODUÇÃO}

Dentre os fatores que norteiam o agricultor, principalmente os que trabalham em regime de sequeiro, o clima é a variável que causa maior influência no rendimento e sustentabilidade econômica das atividades agropecuária (FISHMAN, 2016). Períodos de seca, altas temperaturas, ou chuvas excessivas podem proporcionar danos às lavouras e criações ocasionando baixas produções (LESK et al., 2016). Dessa forma, ter o conhecimento das características climatológicas regionais é uma ferramenta indispensável para uma eficiente gestão de risco visando determinar condições favoráveis de cultivo e criação nas diversas etapas que envolvem a atividade produtiva em campo (HARVEY et al., 2014).

Dentre as variáveis climáticas, a precipitação pluviométrica é a componente que mais condiciona as tomadas de decisões, e que apresenta poucas opções para contornar um excesso ou escassez de água em sistemas de produção de sequeiro (RAY et al., 2015). Esse fato é agravado em regiões tidas marginais de produção, nas quais há, além da ausência ou baixa densidade de estudos, apresentam condições biofísicas menos adequadas de produção e, portanto, de alto risco (ARIAS et al., 2015; PEÑA-GALLARDO et al., 2019). Dentre essas, pode-se citar as novas fronteiras agrícolas situadas na região do MATOPIBA, uma região composta pelos estados do Maranhão, Tocantins, Piauí e Bahia, caracterizada por possuir extensas áreas exploradas pela agropecuária em solos de textura arenosa e um volume pluviométrico razoável, mas que não é bem distribuído ao logo do ano, predominando períodos de estiagem e altas temperaturas (ARIAS et al., 2015).

Em condições de solos arenosos, o balanço hídrico é prejudicado devido a uma menor capacidade de retenção de água nessas classes de solos, maiores taxas de infiltração e percolação líquida (BOHARA et al., 2019), o que gera perdas de água para fora do alcance das plantas prejudicando-as em períodos de ausência de precipitação. Alia-se ao fato que nessas regiões, predominam sistemas convencionais de produção com baixo aporte de palhada, ao contrário do verificado em sistemas conservacionistas do solo e água como o sistema plantio direto e integração lavoura pecuária e floresta. Nesses sistemas a presença de cobertura vegetal viva ou morta (palhada) modifica a dinâmica de água no solo permitindo maior eficiência do uso da mesma pela vegetação (BOHARA et al., 2019; JIA et al., 2019) . Agrega-se que na região do MATOPIBA, localizada entre os paralelos $0^{\circ} \mathrm{e}$ $20^{\circ}$, assiste-se à uma elevada evapotranspiração derivada de maior temperatura do ar e radiação solar (PEREIRA et al., 2018).

Uma ferramenta constantemente utilizada por autores para descrever a chuva em termos de quantidade e de ocorrência é o ajuste de modelos probabilísticos para séries temporais (SILVA et al., 2015; RODRIGUES; THEBALDI, 2016). A modelagem tem por objetivo a aplicação de um modelo matemático, determinístico ou probabilístico, que melhor se ajuste ao comportamento observado de determinado fenômeno, a fim de predizer ou demonstrar a probabilidade de 
ocorrência deste fenômeno (VRAC; NAVEAU, 2008; KOTOWSKI; KAŹMIERCZAK, 2013).

O presente trabalho teve como objetivo analisar os volumes pluviométricos mensais e a distribuição ao logo do ano na região de Formoso, Minas Gerais, por meio de modelagem probabilística e análise descritiva a cada mês.

\section{MATERIAL E MÉTODOS}

Os dados foram obtidos pela estação climática da cidade de Formoso-MG (Latitude: -14.93 Longitude: -46.25), no período de 1977 a 2017, dados corrompidos e falhas de coleta foram excluídos da série (1981, 1982 e de 1988 a 1992), totalizando 33 anos de informação de precipitação pluviométrica.

Localizada na Mesorregião Noroeste de Minas Gerais, Formoso tem clima classificado como AW, de acordo com sistema de classificação climático de Koppen, indicando clima tropical de savana com estação seca de inverno. Já o sistema classificatório de Thornthwaite aponta a cidade como C2wA'a', indicando tipo climático como subúmido, deficiência hídrica moderada no inverno e fator térmico tido como "megatérmico" (MARTINS et al., 2018).

Utilizaram-se de técnicas de modelagem para descrever a distribuição e a variabilidade dos volumes médios mensais precipitados durante o ano. Para o estudo das frequências volumétricas de cada mês foram aplicadas rotinas de modelagem e inferência estatísticas, com o objetivo de obter o ajuste de modelos probabilísticos e selecionando aquele que melhor representaram os dados observados para o mês analisado.

Foram testados ajustes dos modelos exponencial, gama, weibull e log-normal, os parâmetros de cada modelo foram estimados pelo método da máxima verossimilhança. A aderência dos respectivos modelos foi aferida pelo teste de Kolmogorov Smirnov, no caso do ajuste de mais de um modelo foi utilizado o critério de informação de Akaike (AIC) selecionando aquele com menor resultado.

\section{Modelo exponencial}

Uma variável aleatória contínua tem a densidade de probabilidade expressa pela função exponencial para todo valor de $x>0$, quando a fd é dada por:

$$
f(x)=\beta e^{-\beta x}
$$

Sua função cumulativa de densidade (FDP) expressa por:

$$
F(x)=1-e^{-\beta x}
$$

O estimador do parâmetro de escala $\beta$ é dado por:

$$
\hat{\beta}=\frac{n}{\sum_{i=1}^{n} x_{i}}
$$

\section{Modelo gama}

Uma variável aleatória contínua tem a densidade de probabilidade expressa pela função gama para todo valor de $x>0$, quando a fd é dada por: 


$$
f(x)=\frac{\beta^{\alpha} x^{\alpha-1}}{\Gamma(\alpha)} e^{-\beta x}
$$

(4)

Tendo sua FDP dada por:

$$
F(x)=\frac{\gamma(\alpha, \beta x)}{\Gamma(\alpha)}
$$

(5)

Estimadores de parâmetros:

$$
\alpha=\frac{\beta-\sum_{i=0}^{n} x_{i}}{n}
$$

$$
\not=n \alpha+\sum_{i=0}^{n} x_{i}
$$

\section{Modelo weibull}

Uma variável aleatória contínua tem a densidade de probabilidade expressa pela função weibull para todo valor de $x>0$, quando a fd é dada por: $f(x)=\theta \lambda(\lambda x)^{\theta-1} e^{-(\lambda x)^{\theta}}$

Tendo sua FDP dada por:

$$
f(x)=1-e^{-(\lambda x)^{\theta}}
$$

(9)

Estimadores de parâmetros:

$$
\hat{\lambda}=\left(\frac{1}{n} \sum_{i=0}^{n} x_{i}^{\hat{\theta}}\right)^{-\frac{1}{\hat{\theta}}}
$$

$$
\hat{\theta}=\left[\left(\sum_{i=0}^{n} x_{i}^{\hat{\theta}} \log x_{i}\right)\left(\sum_{i=0}^{n} x_{i}^{\hat{\theta}}\right)^{-1}-\frac{1}{n} \sum_{i=0}^{n} \log x_{i}\right]^{-1}
$$

$(10,11)$

\section{Modelo log-normal}

Uma variável aleatória contínua tem a densidade de probabilidade expressa pela função log-normal para todo valor de $x>0$, quando a fd é dada por: $f(x)=\frac{1}{x \sigma \sqrt{2 \pi}} e^{\left[-\frac{(\log x-\mu)^{2}}{2 \sigma^{2}}\right]}$

Tendo sua FDP dada por: 
$f(x)=\phi\left(\frac{\log x-\mu}{\sigma}\right)$

onde $\phi($.$) representa distribuição normal.$

Estimadores de parâmetros:

$\mu=\frac{1}{n} \sum_{i=0}^{n} \log x_{i}$

$b^{2}=\frac{1}{n} \sum_{i=0}^{n}\left(\log x_{i}-\frac{1}{n} \sum_{i=0}^{n} \log x_{i}\right)^{2}$

Os procedimentos foram realizados por meio do software $\mathrm{R}$ com auxílio do pacote fitdistrplus (DELIGNETTE-MULLER; DUTANG, 2015).

\section{RESULTADOS E DISCUSSÃO}

Foi observada na série histórica uma precipitação anual média de $1.198 \mathrm{~mm}$, com desvio padrão de $250 \mathrm{~mm}$. O ano de 1978 apresentou o maior volume de chuvas, de $1880 \mathrm{~mm}$; enquanto em 2007 ocorreram os valores mais baixos $(794 \mathrm{~mm})$ (Figura 1). Mensalmente ocorre uma média de $100 \mathrm{~mm}$, porém as maiores médias são concentradas no período entre novembro e março $(195 \mathrm{~mm})$, com valores praticamente nulos de precipitação nos meses de maio a setembro $(7,4 \mathrm{~mm})$.

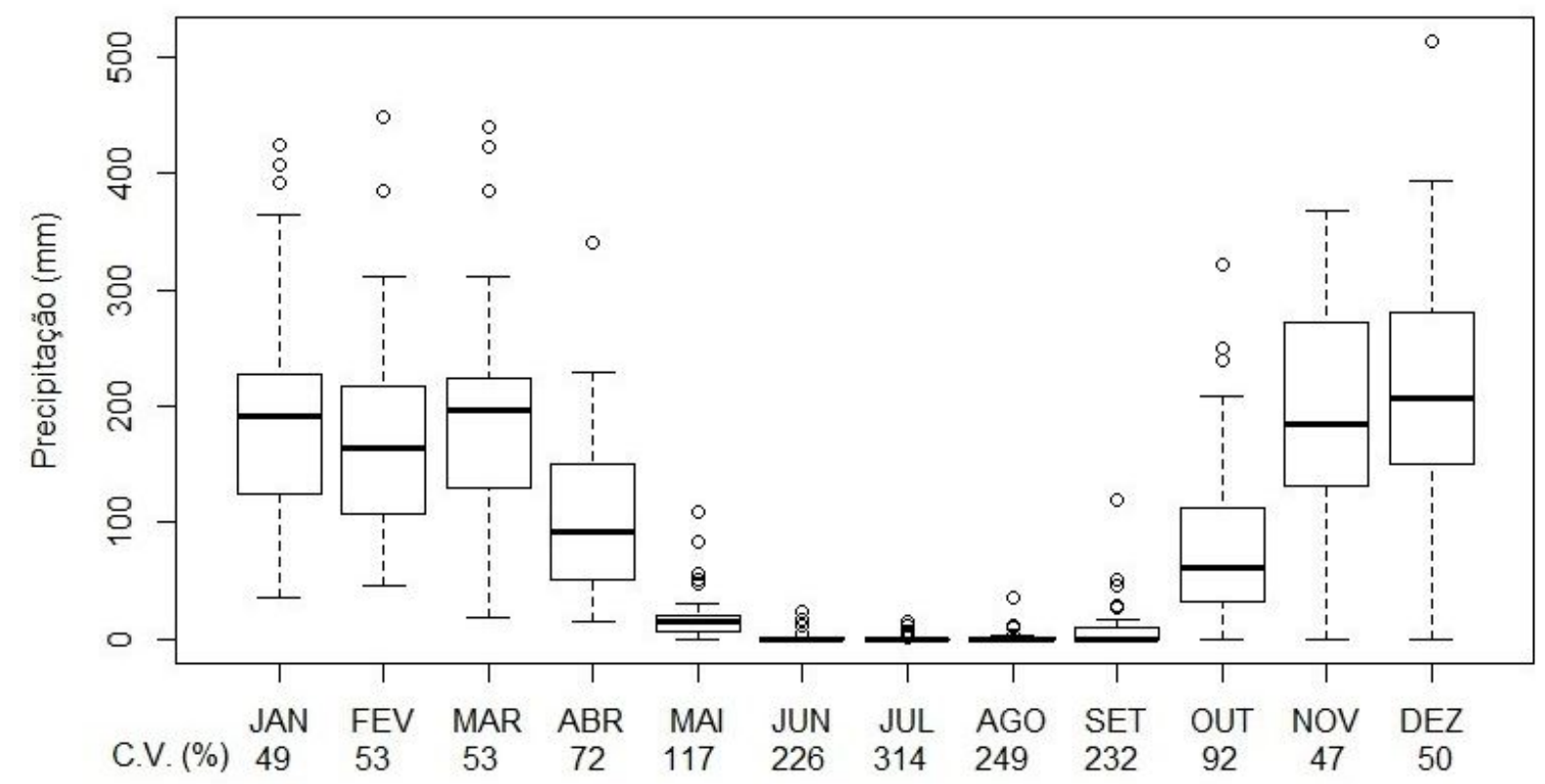

FIGURA 1: Precipitação mensal Formoso, Minas Gerais no período 1977-2017 Fonte: autores.

O período chuvoso tem início em outubro ainda com pequenos volumes e finda em abril, também com chuvas pouco representativas, os maiores volumes são dispostos entre novembro e março totalizando uma média de $974 \mathrm{~mm}$, representando 
$81 \%$ da chuva anual (Figura 1). Os demais meses, período entre maio e setembro, são os meses de seca com 3\% da precipitação anual distribuídos entre os mesmos.

Em sistemas de sequeiro, o adequado regime hídrico pluviométrico é preponderante para evitar situações de estresse hídrico e, portanto, efeitos negativos sobre o crescimento e desenvolvimento vegetal das culturas durante o seu ciclo. A oferta hídrica local média observada neste trabalho, no período de safra verão, entre os meses de novembro e fevereiro foi de $783 \mathrm{~mm}$. Volume que atende à demanda hídrica de várias culturas, tal como o milho (Zea mays L.), que tem necessidade hídrica entre 500 e $800 \mathrm{~mm}$, soja (Glycine max L.) entre 450 e $850 \mathrm{~mm}$ e arroz (Oryza sativa L.) com necessidade entre 450 e $700 \mathrm{~mm}$ (BONETTI et al., 2017). Contudo, deve-se observar que, pela análise dos anos, verificou-se anos que tiveram ausência completa de precipitação nos meses de maio até dezembro. Mesmo em frequência baixa, tais cenários geram, aliadas às condições de baixa retenção de água no solo e práticas de revolvimento, um moderado risco no cultivo de lavouras no período (Figura 1).

Não somente o volume total precipitado durante o ciclo deve ser considerado, assim como a adequada distribuição desde o plantio à colheita. Na cultura do milho, que apresenta ciclo que varia de 110 a 180 dias de acordo com a cultivar, verificamse sérias reduções de produtividade quando há escassez de água no período que antecede a emissão das anteras e no florescimento (BERGAMASCHI et al., 2006). Portanto, no que tange decisões para implantação de culturas, deve-se observar além da necessidade em cada fase fenológica, outros fatores climáticos, tais como evapotranspiração, retenção hídrica do solo e fotoperíodo (TEIXEIRA et al., 2017).

De fevereiro a maio verifica-se um total de $491 \mathrm{~mm}$ (Figura 1). Considerando que o solo nesse período de fevereiro apresenta boa disponibilidade de água devido ao período anterior de alta precipitação média, aponta-se que é viável a implantação de culturas que promovam uma boa cobertura do solo e geração de palhada (TERAVEST et al., 2015). Espécies gramíneas que apresentam boa características recalcitrantes como as do gênero das braquiárias, são opções notáveis para a implantação na entressafra, período que contempla o ciclo destas espécies, evitando exposição e melhorando a nutrição orgânica do sistema solo (AKER; PASSOS., 2018).

Dentre os modelos que tiveram a aderência testada, o modelo gama teve representatividade para os meses de janeiro, fevereiro e abril, o modelo weibull obteve sucesso para os meses de novembro e março; ambos se mostraram eficazes no ajuste aos meses do período chuvoso, quando se tem os maiores volumes e variabilidade, já o modelo exponencial ajustou-se aos meses de maio e outubro quando a maior frequência são de valores reduzidos de precipitação (Tabela 01). 
TABELA 1: Parâmetros dos modelos Exponencial, Gama, Weibull e Log-normal, valores de teste de aderência KolmogorovSmirnov e seleção de modelo para série histórica de precipitação de Formoso,

MG.

\begin{tabular}{|c|c|c|c|c|c|c|c|c|c|c|c|c|c|c|c|c|}
\hline \multirow[b]{3}{*}{ MÊS } & \multicolumn{7}{|c|}{ PARÂMETROS } & \multicolumn{4}{|c|}{ Teste de aderência Kolmogorov-Smirnov } & \multicolumn{4}{|c|}{ Critério de seleção de Akaique } & \multirow{3}{*}{$\begin{array}{c}\text { MODELO } \\
\text { SELECIONADO }\end{array}$} \\
\hline & \multirow{2}{*}{$\frac{\text { EXPONENCIAL }}{\beta}$} & \multicolumn{2}{|c|}{ GAMA } & \multicolumn{2}{|c|}{ WEIBULL } & \multicolumn{2}{|c|}{ LOG NORMAL } & \multicolumn{4}{|c|}{ P-Valor } & \multicolumn{4}{|c|}{ AIC ** } & \\
\hline & & $\alpha$ & $\beta$ & $\beta$ & $\alpha$ & $\mu$ & $\sigma$ & Exponencial & Gama & Weibull & Log Normal & Exponencial & Gama & Weibull & Log Normal & \\
\hline JAN & 0,01 & 4,17 & 0,02 & 2,20 & 217,35 & 5,13 & 0,53 & $0,46 \%$ & $78,39 \%$ & $52,76 \%$ & $77 \%$ & 440,10 & 415,53 & 416,33 & 417,99 & Gama \\
\hline FEV & 0,01 & 3,69 & 0,02 & 2,03 & 197,97 & 5,02 & 0,55 & $1,39 \%$ & $99,74 \%$ & $92,33 \%$ & $98 \%$ & 433,44 & 412,31 & 413,73 & 413,27 & Gama \\
\hline MAR & 0,01 & 2,96 & 0,02 & 1,99 & 215,92 & 5,08 & 0,68 & $1,32 \%$ & $35,96 \%$ & $63,77 \%$ & $20 \%$ & 439,96 & 424,72 & 422,62 & 431,44 & Weibull \\
\hline$A B R$ & 0,01 & 1,87 & 0,02 & 1,45 & 115,04 & 4,35 & 0,83 & $31,37 \%$ & $88,52 \%$ & $89,25 \%$ & $43 \%$ & 397,13 & 392,31 & 392,37 & 394,91 & Gama \\
\hline MAI & 0,05 & 0,36 & 0,02 & 0,50 & 14,77 & 1,12 & 4,61 & $55,32 \%$ & $0,20 \%$ & $0,52 \%$ & $0 \%$ & 282,60 & 244,40 & 256,06 & 288,79 & Exponencial \\
\hline JUN & 0,34 & 0,09 & 0,03 & 0,15 & 0,01 & $-7,73$ & 6,01 & $0,00 \%$ & $0,00 \%$ & $0,00 \%$ & $0 \%$ & 146,87 & $-295,15$ & $-301,58$ & $-312,52$ & NA \\
\hline JUL & 0,97 & 0,09 & 0,09 & 0,17 & 0,00 & $-9,32$ & 4,84 & $0,00 \%$ & $0,00 \%$ & $0,00 \%$ & $0 \%$ & 73,97 & $-402,84$ & $-420,56$ & $-438,90$ & NA \\
\hline AGO & 0,37 & 0,10 & 0,04 & 0,16 & 0,02 & $-7,41$ & 6,10 & $0,00 \%$ & $0,00 \%$ & $0,00 \%$ & $0 \%$ & 141,90 & $-274,92$ & $-279,27$ & $-288,64$ & NA \\
\hline SET & 0,10 & 0,10 & 0,01 & 0,15 & 0,07 & $-6,23$ & 6,91 & $0,00 \%$ & $0,00 \%$ & $0,00 \%$ & $0 \%$ & 232,98 & $-191,52$ & $-190,99$ & $-197,34$ & NA \\
\hline OUT & 0,01 & 0,39 & 0,00 & 0,55 & 66,02 & 2,75 & 4,49 & $76,07 \%$ & $0,72 \%$ & $1,87 \%$ & $0 \%$ & 381,63 & 352,57 & 363,71 & 400,67 & Exponencial \\
\hline NOV & 0,01 & 1,04 & 0,01 & 1,37 & 205,85 & 4,74 & 2,82 & $0,31 \%$ & $0,42 \%$ & $6,56 \%$ & $0 \%$ & 442,05 & 444,01 & 440,20 & 507,49 & Weibull \\
\hline DEZ & 0,00 & 1,01 & 0,00 & 1,31 & 226,55 & 4,81 & 2,85 & $0,20 \%$ & $0,21 \%$ & $2,63 \%$ & $0 \%$ & 448,90 & 450,89 & 447,92 & 513,47 & NA \\
\hline
\end{tabular}


Tais observações condizem em parte com o observado por Rodrigues e Thebaldi (2013), em que as densidades de probabilidade das funções gama e weibull, nesta ordem, foram as que melhor se ajustaram, seguidas pelo modelo lognormal que no presente estudo obteve ajuste para os meses de janeiro e fevereiro, porém com menor significância quando comparada a gama e weibull.

Quando utilizados os modelos para análise diária de precipitação as mesmas funções (gama e weibull) mostraram ajuste muito próximos, demonstrando a flexibilidade e eficiência destes modelos para aplicação em diversas séries temporais (SILVA et al., 2015). Em um grande número de situações, o modelo determinístico é suficiente. Contudo, existem muitos fenômenos que requerem um modelo diferente para sua investigação, como o caso do estudo da precipitação de uma determinada região. Observações meteorológicas fornecem informações consideráveis, mas não se faz possível uma predição eficaz (MEYER, 1972); por tanto, um modelo probabilístico se faz mais eficiente, apontando com base na série histórica a probabilidade de ocorrência de determinado volume.

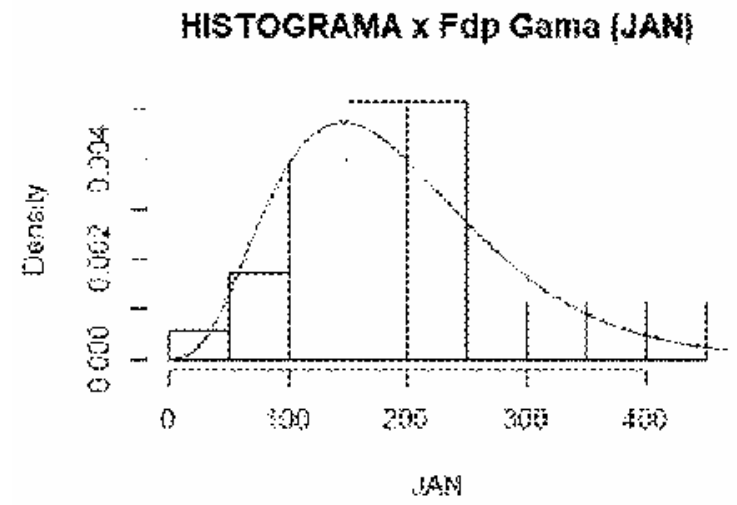

HSTOGRAMA X Fdp WeibUHI (MAR)

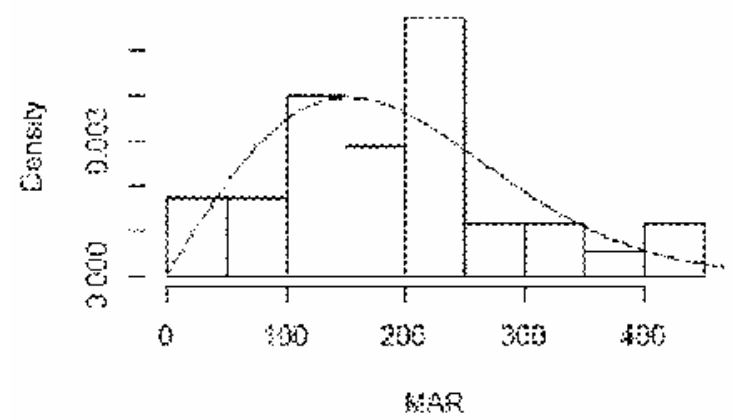

HIS TOGSAMA X Fơ Gana (FEV)

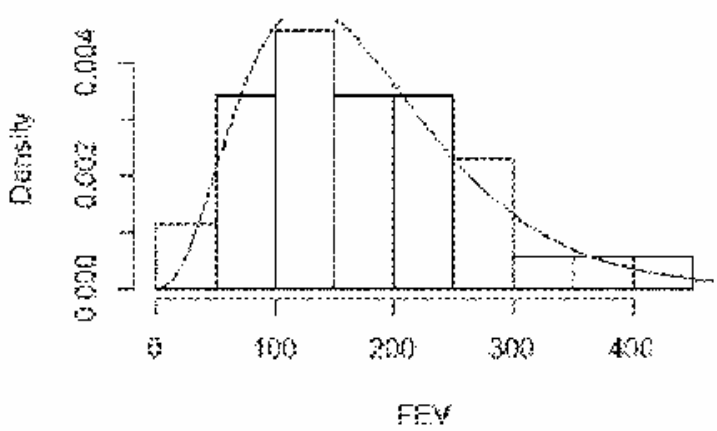

HISTOGRAMA X Fdp Gama (ABR)

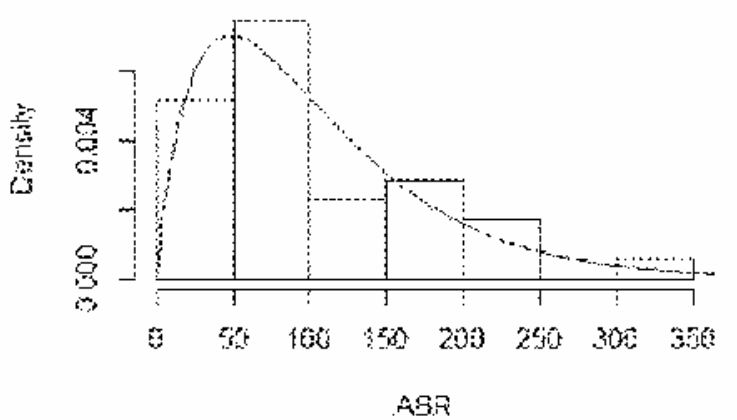



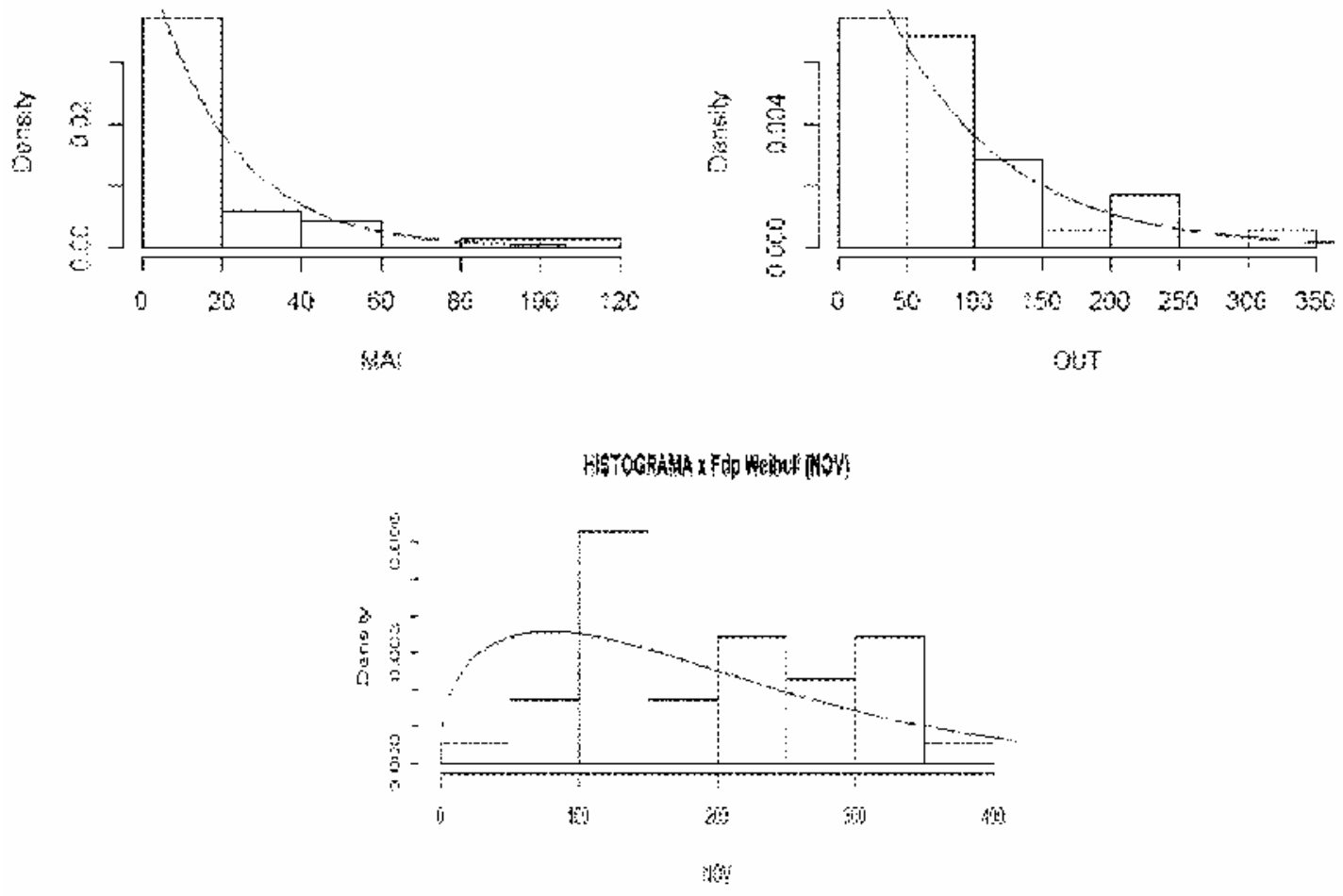

FIGURA 2: Distribuições de frequências volumétricas observadas e ajustes das funções densidade para os meses de janeiro, fevereiro, março, abril, maio, outubro e novembro da série histórica para Formoso, MG. Fonte: autores.

Juntamente com o conhecimento do regime climático regional e das necessidades fisiológicas das culturas, a aplicação de técnicas conservacionistas como plantio direto, rotação de cultura e integração entre lavoura, pecuária e floresta, são opções para evitar a exposição e degradação do solo além de melhorar o aporte de matéria orgânica.

\section{CONCLUSÕES}

Os modelos weibull e gama apresentaram melhores ajustes para os meses que concentram maior volume de precipitação, exceto em dezembro, mês que não houve ajuste de nenhum dos modelos testados.

A distribuição exponencial se mostrou aderente aos períodos que a ocorrência é de baixos volumes de chuva.

\section{REFERÊNCIAS}

AKER, A. M.; PASSOS, A. M. A. dos. Performance de plantas leguminosas para sistema plantio direto na região amazônica. Enciclopédia Biosfera, v. 15, n. 28, p. 804-814, 2018. Disponível em: <http://dx.doi.org/10.18677/EnciBio_2018B66>. Doi: 10.18677/EnciBio_2018B66. 
ARIAS, D.; MENDES, P.; ABEL, P. Revisão rápida e integrada da gestão de riscos agropecuários no Brasil: caminhos para uma visão integrada: 1.ed.Brasília, DF: Banco Mundial, n 76 p., 2015.

BERGAMASCHI, H.; DALMAGO, G.A.; COMIRAN, F.; BERGONCI, J.I.; MÜLLER, A.G.; et al. Deficit hídrico e produtividade na cultura do milho. Pesquisa Agropecuária Brasileira, v.41, p.243-249, 2006. Disponível em: <http://dx.doi.org/10.1590/S0100-204X2006000200008>, doi: 10.1590/S0100204X2006000200008.

BOHARA, H.; DODLA, S.; WANG, J. J.; DARAPUNENI, M.; ACHARYA, B. S. et al. Influence of poultry litter and biochar on soil water dynamics and nutrient leaching from a very fine sandy loam soil. Soil and Tillage Research, v.189, p.45-51, 2019. Disponível em: <https://doi.org/10.1016/j.still.2019.01.001>, doi: 10.1016/S0100204X2006000200008.

BONETTI, J. A.; ANGHINONI, I.; MORAES, M. T.; FINK, J. R. Resilience of soils with different texture, mineralogy and organic matter under long-term conservation systems. Soil and Tillage Research, v. 174, p. 104-112, 2017. Disponível em: <http://dx.doi.org/10.1016/j.still.2017.06.008> doi: 10.1016/j.still.2017.06.008.

DELIGNETTE-MULLER, M.L; CHRISTOPHE DUTANG; An R Package for Fitting Distributions. Journal of Statistical Software, 64(4), 1-34. Disponível em < http://www.jstatsoft.org/v64/i04/. > 2015.

FISHMAN, R. More uneven distributions overturn benefits of higher precipitation for crop yields. Environmental Research Letter v.11, n.2, p.024004, 2016. Disponível em: $\quad<$ https://doi.org/10.1088/1748-9326/11/2/024004>,doi:10.1088/1748$9326 / 11 / 2 / 024004$

HARVEY, C. A.; CHACÓN, M.; DONATTI, C.I.; GAREN, E.; HANNAH, L.; et al. Climate-smart landscapes: opportunities and challenges for integrating adaptation and mitigation in tropical agriculture. Conservation Letter. 7:77-90. Disponível em: doi.10.1111/conl.12066, 2014.

JIA, L.; ZHAO, W.; FU, B.; SARYANTO, S.W.; WANG, S. et al. Effects of minimum soil disturbance practices on controlling water erosion in China's slope farmland: A meta-analysis. Land Degradation and Development. 2019; 30: 706- 716. https://doi.org/10.1002/ldr.3258

KOTOWSKI, A.; KAŹMIERCZAK, B.; Probabilistic models of maximum precipitation for designing sewerage. Journal Hydrometeor. 14(6):1958-1965, 2013. Disponível em: $<$ https://doi.org/10.1080/19475705.2019.1585968.> doi 10.1080/19475705.2019.1585968.

LESK, C.; ROWHANI, P.; RAMANKUTTY, N. Influence of extreme weather disasters on global crop production. Nature 529(7584):84-87, 2016. Disponível em: $<$ https://doi.org/10.1080/19475705.2019.1585968.> doi 10.1080/19475705.2019.1585968. 
MARTINS, F.; GONZAGA, G.; SANTOS, D.; REBOITA, M. Classificação climática de Köppen e de Thornthwaite para Minas Gerais: cenário atual e projeções futuras. $\begin{array}{lllll}\text { Revista Brasileira de } & \text { Climatologia. v.1, p129-156. }\end{array}$ http://dx.doi.org/10.5380/abclima.v1i0.60896

MEYER, L.P. Probabilidade: aplicações à estatística. Rio de Janeiro, Ao Livro Técnico S. A, 1972.

PEÑA-GALLARDO, M.; VICENTE-SERRANO, S. M.; QUIRING, S.; SVOBODA, M.; HANNAFORD, J.; et al. Response of crop yield to different time-scales of drought in the United States: Spatio-temporal patterns and climatic and environmental drivers. Agricultural and Forest Meteorology. v. 264, p. 40-55, 2019.

PEREIRA, C.N.; CASTRO, C.N.D.; PORCIONATO, G.L. Dinâmica econômica, infraestrutura e logística no Matopiba, Brasília, Instituto de Pesquisa Econômica Aplicada- IPEA, 2018.

RAY, D.K.; J.S. GERBER, G.K. MACDONALD, P.C. West Climate variation explains a third of global crop yield variability, Nature communications | 6:5989 | DOI: 10.1038/ncomms6989 | 2015. Disponível em: DOI: 10.1038/ncomms6989

RODRIGUES, G. M. R.; THEBALDI, M, S.; Estimativa da precipitação máxima diária anual e equação de chuvas intensas para o município de Formiga, MG, Brasil. Revista Ambiente \& Água. v. 11 n. 4, p.891-902 Disponível em: $<$ https://doi.org/10.4136/ambi-agua.1823, 2016.> doi:10.4136/ambi-agua.1823, 2016.

SILVA, D. A. A.; SANTI, A. L.; DAL BELLO, R. A. M; FIORESI, D.; BARON, F. A. Perfil de NDVI em estádios de desenvolvimento de soja em função de diferentes plantas de coberturas do solo no Rio Grande do Sul. Enciclopédia Biosfera, v. 11, n. 22, p. 2340-2348, 2015.2015 Disponível em: <https://doi.org/10.18677/Enciclopedia_Biosfera_2015_205.> doi 10.18677/Enciclopedia_Biosfera_2015_205

TEIXEIRA, P.C.; DONAGEMMA, G.K.; FONTANA, A.; TEIXEIRA, W. G. Manual de métodos de análise de solo. Brasília-DF, 2017.

TERAVEST, D.; CARPENTER-BOGGS, L.; THIERFELDER, C.; REGANOLD, J. P.; Crop production and soil water management in conservation agriculture, no-till, and conventional tillage systems in Malawi. Agriculture, Ecosystems \& Environment, v. 212, n. 20, p. 285-296, 2015. Disponível em: <https://doi.org/10.1016/j.agee.2015.07.011.> doi: 10.1016/j.agee.2015.07.011.

VRAC, M.; NAVEAU, P. "Stochastic downscaling of precipitation: From dry events to heavy rainfall" Water Resources Research. Volume 44lssue 5 2008. Disponível em: < https://doi.org/10.1029/2006WR005308>,doi:10.1029/2006WR005308. 